\title{
Age-dependent Excystment of the Protozoan Acanthamoeba castellanii
}

\author{
By J. A. CHAMBERS AND J. E. THOMPSON \\ Department of Biology, University of Waterloo, Waterloo, Ontario, Canada
}

(Received I4 May I973; revised I3 August 1973)

\begin{abstract}
SUMMARY
Axenic cyst cultures of Acanthamoeba castellanii (Neff strain) maintained in salt medium undergo excystment when transferred into enriched growth medium containing yeast extract and proteose peptone. Both the synchrony and extent of excystment induced in this manner are critically dependent upon the length of time cysts are aged in salt medium prior to the initiation of excystment. Cysts sustained in salt medium for $50 \mathrm{~h}$ or less characteristically show only about I0 \% excystment even after $70 \mathrm{~h}$ in the enriched growth medium. With progressively longer periods of ageing the synchrony and degree of excystment improve such that for cysts of age $\geqslant 800 \mathrm{~h}$, more than $85 \%$ of the organisms germinate within a period of $\mathrm{I} 2 \mathrm{~h}$. Thus more complete and relatively synchronous excystment can be achieved by this simple experimental manipulation.
\end{abstract}

\section{INTRODUCTION}

Acanthamoeba castellanii, like many protozoa, adapts to alternating favourable and unfavourable environmental conditions by undergoing reversible differentiation. It encysts under environmental stress and excysts once propitious conditions return. The trophozoites (amoebae) can be cultured axenically in an enriched growth medium containing yeast extract and proteose peptone and synchronously encyst when transferred to an appropriate salt medium devoid of organic nutrients (Neff, 1957; Neff, Ray, Benton \& Wilborn, I964 b). Excystment is induced by simply placing the cysts back in the enriched growth medium. However, the usefulness of this procedure in experimental investigations of excystment is limited by the fact that it gives rise to incomplete and highly asynchronous germination (Mattar \& Byers, 197I ; Chambers \& Thompson, 1972).

Structural, chemical and functional changes accompanying encystment of Acanthamoeba have been documented (Neff, I957; Tomlinson \& Jones, 1962; Tomlinson, 1963; Neff et al. I964 $b$; Neff, Benton \& Neff, I964; Tomlinson, 1967; Bowers \& Korn, I969; Pasternak, Thompson, Schultz \& Zachariah, 1970), and this is partly because procedures for inducing relatively synchronous encystment are available. Excystment has been much less extensively examined. Information about the sequence of the structural and morphological changes the organism undergoes as it excysts has been obtained by phase-contrast microscopy (Mattar \& Byers, 1971) and scanning electron microscopy (Chambers \& Thompson, 1972). This was possible despite asynchrony of the excysting cultures because organisms representative of particular stages of differentiation could be recognized in heterogeneous populations. Parallel studies of functional and chemical changes are virtually impossible under these conditions, however, because large homogeneous populations of organisms representative of each definitive stage of excystment are needed. During an investigation of ways to surmount this problem we found that capability for excystment is clearly related to cyst age. 
When encystment is induced in a salt medium originally formulated for this purpose by Neff et al. (1964b), synchronous emergence is possible only if the cysts are aged in the salt medium for a period of time before excystment is initiated.

\section{METHODS}

Vegetative cells of Acanthamoeba castellanii were cultured at $29{ }^{\circ} \mathrm{C}$ in $250 \mathrm{ml}$ Erlenmeyer flasks containing $50 \mathrm{ml}$ of the enriched growth medium originally defined for this organism by Neff et al. (1964b). Aeration was achieved by shaking at Ioo rev./min.

Encystment and excystment were obtained by procedures described previously (Chambers \& Thompson, 1972). The method for encystment was essentially the same as that formulated originally by Neff et al. (I964b) except that aeration was achieved by rotating the flasks at roo rev./min. After $48 \mathrm{~h}$ at least $95 \%$ of the organisms had encysted as judged by phasecontrast microscopy. At this stage the cultures were removed from the shaker and left stationary at $29^{\circ} \mathrm{C}$ for additional periods of up to $\mathrm{I} 600 \mathrm{~h}$ before being used for excystment. To provide adequate aeration, the stationary cultures were swirled by hand once every 2 days. Cysts derived from $250 \mathrm{ml}$ of encystment medium were resuspended in $125 \mathrm{ml}$ of enriched growth medium, placed in a $250 \mathrm{ml}$ Erlenmeyer flask and maintained at $29^{\circ} \mathrm{C}$ for $70 \mathrm{~h}$ or until excystment was complete. Aeration was achieved by shaking the excysting cultures at I00 strokes/min on an Eberbach reciprocating shaker. Particular care was taken to prevent the cysts being sucked dry during harvest from the encystment medium by millipore filtration. This precaution minimized clumping in the excysting cultures.

Excystment was monitored by examining $3 \mathrm{ml}$ samples taken sequentially from excysting cultures. The first sample was taken immediately after transfer of the mature cysts into the optimal growth medium. Subsequent samples were collected at intervals depending on the rapidity of excystment. For each sample, the organisms were centrifuged at $800 \mathrm{~g}$ for $2 \mathrm{~min}$ and resuspended in the iodine-eosine stain described by Mattar \& Byers (197I). Wet mounts of the stained organisms were examined by phase-contrast microscopy and at least 500 cysts and empty cyst walls combined were identified and counted. The extent of excystment for each sample was expressed as

$$
\text { Percentage excystment }=\frac{\text { empty cyst walls }}{\text { empty cyst walls }+ \text { cysts }} \times 100 .
$$

Sometimes large clumps of cysts were present in the collected samples and these were dispersed by hand homogenization with one stroke of a loose-fitting pestle-type homogenizer. The cells were then centrifuged and resuspended in stain according to the routine procedure.

\section{RESULTS AND DISCUSSION}

Measurements of the numbers of empty cyst walls in excysting cultures proved reliable for determining the rate of excystment. The cyst walls persist as recognizable structures for at least $75 \mathrm{~h}$ following emergence of the trophozoite (Mattar \& Byers, I97I) and are readily distinguishable from intact cysts and amoebae by phase-contrast microscopy, particularly after staining with iodine-eosine. Some breakage of cysts ( 2 to $4 \%$ ) occurred as a result of the gentle homogenization used to disperse clumps. However, it was possible to calculate the extent to which this was occurring for any one culture by determining the number of empty cyst walls in the first sample taken at zero time before the cysts were transferred into the excystment medium; any empty cyst walls in this sample could only be a manifestation 

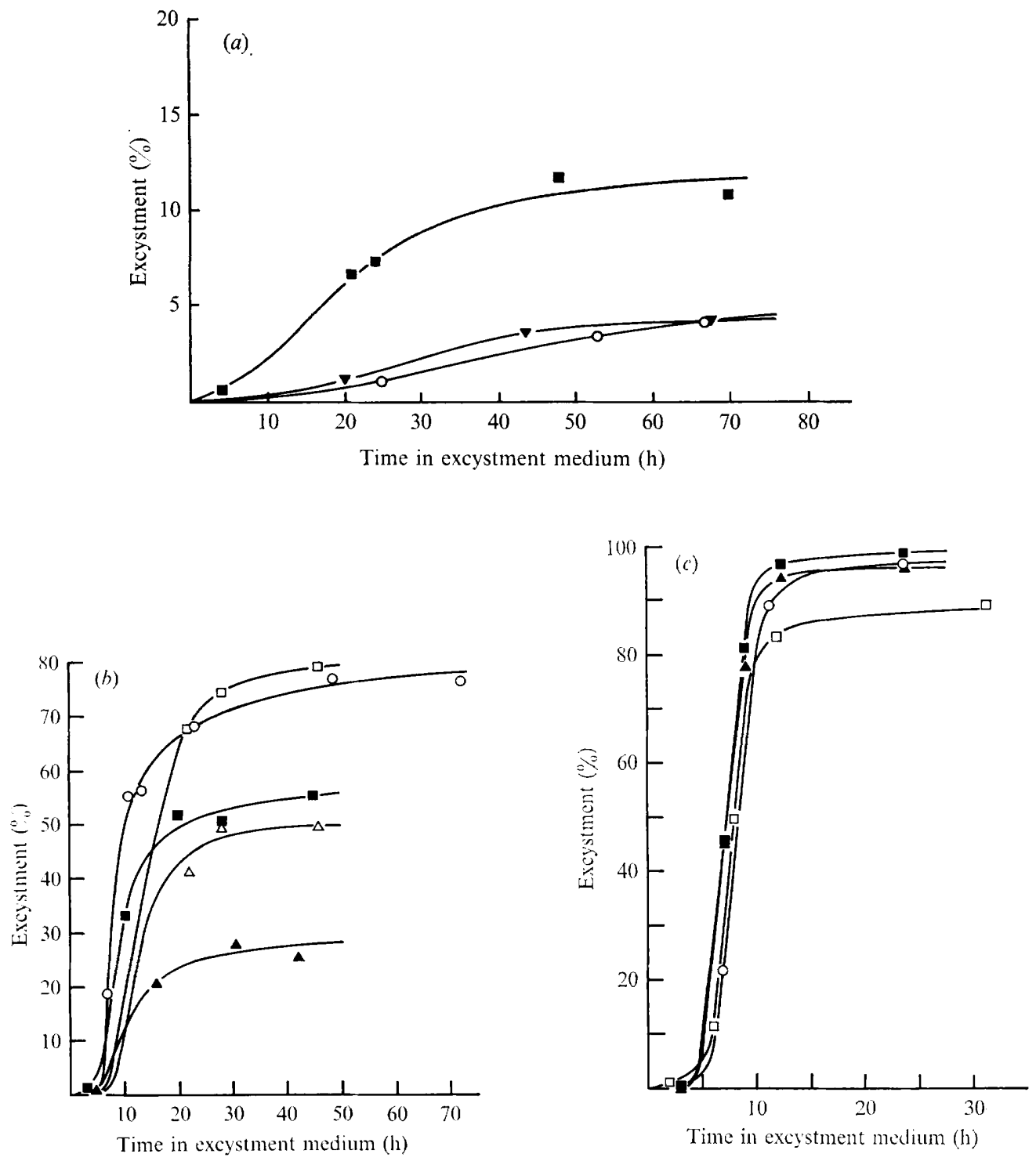

Fig. I. Excystment of Acanthamoeba castellanii. Each point on the profiles was obtained by counting at least 500 cysts and empty cyst walls. The profiles represent separate experiments with different cultures of cysts, except for the two $\mathrm{I} 605 \mathrm{~h}$ profiles which are separate excystments from the same cyst culture. (a) Cysts less than $50 \mathrm{~h}$ of age. $\mathbf{7}, 29 \mathrm{~h} ; \mathrm{O}, 30 \mathrm{~h} ; \boldsymbol{m}, 49 \mathrm{~h}$. (b) Cysts ranging in age from $70 \mathrm{~h}$ to about $500 \mathrm{~h} . \triangle, 73 \mathrm{~h} ; \Delta, \mathrm{I} 7 \mathrm{I} \mathrm{h} ; \mathrm{m}, \mathrm{I} 9 \mathrm{I} \mathrm{h} ; \mathrm{O}, 500 \mathrm{~h} ; \square, 50 \mathrm{I} \mathrm{h} .(\mathrm{c})$ Cysts ranging in age from $800 \mathrm{~h}$ to about I $600 \mathrm{~h} . \mathrm{O}, 887 \mathrm{~h} ; \square, \mathrm{I} 460 \mathrm{~h} ; \mathbf{\Delta}$ and $\mathrm{E}, \mathrm{I} 605 \mathrm{~h}$.

of cyst breakage. The percentage breakage so obtained was subtracted as a correction factor from the calculated percentage excystment for those samples requiring dispersal of cyst clumps. No significant difference was evident between experiments in which clumps of cysts needed to be dispersed by homogenization and those in which this was not necessary.

Rates of excystment for cultures aged in the encystment salt medium for varying periods of time are illustrated in Fig. I. These results are representative of those obtained from at 
least 50 separate experiments. In general, with increased length of the ageing period there is a corresponding increase in both the synchrony and the extent of excystment. Cultures 30 to $50 \mathrm{~h}$ of age are capable of only about $10 \%$ excystment, and this occurs essentially asynchronously (Fig. I $a$ ). By $500 \mathrm{~h}$ a much higher proportion of the organisms is able to excyst and some degree of synchrony is evident (Fig. I $b$ ). After $800 \mathrm{~h}, 85$ to $95 \%$ of the organisms excyst within about $\mathrm{I} 2 \mathrm{~h}$ of the initial transfer of cysts into the growth medium (Fig. I c). In all experiments the emergence scored was a reflexion of true germination and not cyst breakage, as shown by the fact that exit holes could be seen on the empty cyst walls. Scanning electron microscopy reveals that these holes comprise an ostiole with its operculum removed (Chambers \& Thompson, 1972). When gentle homogenization was used to disperse cyst clumps, the few cyst walls arising as a result of cyst breakage were recognizable as such since they retained remnants of cytoplasm and often looked crushed.

Older cyst cultures display a more synchronous and more complete excystment, although there is some overlapping in that for cultures with relatively small differences in age, the older culture does not always yield the higher percentage excystment. For example, in Fig. I $b$ the culture aged for $73 \mathrm{~h}$ excysts more completely and rapidly than that aged for I $7 \mathrm{~h}$. This variation presumably reflects innate differences among cultures of cysts rather than experimental and sampling variance, since different excystments prepared from the same cyst culture yielded closely similar emergence profiles (e.g. the two I605 h experiments of Fig. I c).

Oxygen is obligatory for encystment in salt medium (Neff \& Neff, 1969). In our study, this requirement was met by shaking the encysting organisms until encystment was complete. Mature cysts are essentially dormant and their respiration rates are very low. Indeed, oxygen consumption by cysts of Acanthamoeba castellanii a week or more old is not detectable by Warburg respirometry (Neff \& Neff, 1969). Accordingly, in this study once mature cysts had formed, the cultures were left stationary during subsequent ageing in the encystment salt medium and shaken by hand only intermittently. The cysts retained their round and wrinkled forms during this ageing and also remained viable as judged by their appearance under a phase-contrast microscope. Consistently, after more than $\mathrm{I} 600 \mathrm{~h}$ of saline incubation fewer than $\mathrm{I} \%$ of the organisms had lost the refractility that normally characterizes a living cyst.

Several protozoa, including Didinium nasutum (Butzel \& Horwitz, 1965), Tillina magna (Beers, I945), Blepharisma stoltei (Repak, 1968) and Schizopyrenus russelli (Singh, Mathew \& Anand, I958), require exposure to an environment containing bacteria before effectively excysting. For $S$. russelli, aqueous extracts from Aerobacter sp. or Escherichia coli will induce quite synchronous emergence (Singh, Saxena \& Iyer, 1965; Singh, Datta \& Dutta, 197I; Rastogi, Sagar \& Agarwala, 197I). These experiments were carried out in nonnutrient medium supplemented with the extracts. The components of the extracts primarily responsible for the excystment-inducing activity appeared to be amino acids. We tested the effects of Aerobacter sp. and of the aqueous extracts described by Singh et al. (1965, I97I) on the excystment of young cyst cultures of Acanthamoeba castellanii, but to no avail. Neither the extracts nor the bacteria themselves influenced the rate of emergence. This is perhaps not surprising, however, for amino acids, the putative active components of the extracts, are abundantly present in the enriched growth medium we used for excystment.

From the results shown in Fig. I, mere exposure of newly matured cysts of Acanthamoeba castellanii to a renewed source of nutrient is apparently not sufficient to spark excystment. Cysts which look mature are formed after only $24 \mathrm{~h}$ in encystment medium, yet organisms which have been in this medium for as long as $50 \mathrm{~h}$ are still for the most part incapable of 
excysting when transferred to the enriched growth medium. This suggests that some metabolic or physiological process, initiated when encystment begins, must run its full course irrespective of the presence of nutrients before excystment will occur. This process may constitute a final phase of cyst maturation that is not manifested morphologically and thus has hitherto escaped notice. Given that this is the case, the absence of a sharp threshold during ageing of the organisms in encystment medium, beyond which excystment is complete and synchronous and below which it is incomplete and asynchronous, implies that this aspect of cyst development is not well synchronized in cultures encysted according to the procedure of Neff et al. (I964b). Thus it is only after the organisms have been in the encystment medium for more than $800 \mathrm{~h}$ that synchronous and complete excystment can be obtained.

The poor degree of excystment evident in asynchronous cultures (Fig. I $a$ ) may be attributable to an excystment inhibitor produced by the few trophozoites that emerge and begin to replicate. According to Dubes \& Jensen (I964), such an inhibitor does accumulate in actively growing cultures of Acanthamoeba trophozoites in concentrations directly proportional to the cell population density. There is sufficient time in asynchronous cultures with low excystment rates for trophozoites significantly to increase their numbers by replication, but this does not hold true for synchronous cultures. Thus, the inhibitor would be expected to have a pronounced effect on young cultures and virtually no effect on synchronously excysting cultures.

The cellular basis for the ageing in salt medium that appears to be a prerequisite for successful excystment of Acanthamoeba castellanii remains unsolved. This notwithstanding, these observations demonstrate that by a very simple experimental manipulation, cyst cultures can be induced to undergo synchronous and essentially complete excystment. This opens the way for a comprehensive examination of the functional changes that accompany germination of this organism.

This work was supported by the National Research Council of Canada.

\section{REFERENCES}

BEERs, C. D. (1945). Some factors affecting excystment in the ciliate Tillina magna. Physiological Zoölogy 18, 80-99.

Bowers, B. \& KORN, E. D. (1969). The fine structure of Acanthamoeba castellanii (Neff strain). II. Encystment. Journal of Cell Biology 4r, 786-805.

Butzel, H. M., Jun. \& Horwitz, H. (1965). Excystment of Didinium nasutum. Journal of Protozoology 12, $413-416$.

Chambers, J. A. \& Thompson, J. E. (1972). A scanning electron microscopic study of the excystment process of Acanthamoeba castellanii. Experimental Cell Research 73, 41 5-421.

DubEs, G. R. \& JENSEN, T. (1964). Production and properties of an excystment inhibitor from Acanthamoeba sp. Journal of Parasitology 50, 380-385.

MATtAR, F. E. \& BYERS, T. J. (I971). Morphological changes and the requirements for macromolecule synthesis during excystment of Acanthamoeba castellanii. Journal of Cell Biology 49, 507-519.

NefF, R. J. (1957). Purification, axenic cultivation and description of a soil amoeba Acanthamoeba sp. Journal of Protozoology 4, I76-I 82.

NefF, R. J., Benton, W. F. \& NeFF, R. H. (Ig64a). The composition of the mature cyst wall of the soil amoeba Acanthamoeba sp. Journal of Cell Biology 23, $66 \mathrm{~A}$.

NefF, R. J., Ray, S. A., Benton, W. F. \& Wilborn, M. (1964b). Induction of synchronous encystment in Acanthamoeba sp. In Methods in Cell Physiology, pp. 55-83. Edited by D. M. Prescott. New York: Academic Press.

NefF, R. J. \& NeFF, R. H. (1969). The biochemistry of amoebic encystment. In Dormancy and Survival, Symposia of the Society for Experimental Biology, pp. 5I-8I. Cambridge University Press. 
Pasternak, J. J., Thompson, J. E., Schultz, T. M. G. \& Zachariah, K. (I970). A scanning electron microscopic study of the encystment of Acanthamoeba castellanii. Experimental Cell Research 60, $290-298$.

Rastogi, A. K., Sagar, P. \& Agarwala, S. C. (197r). Changes in the levels of some macromolecules and certain enzyme activities in excysting Schizopyrenus russelli. Journal of Protozoology 18, 506-509.

REPAK, A. J. (1968). Encystment and excystment of the heterotrichous ciliate Blepharisma stoltei Isquith. Journal of Protozoology r5, 407-412.

Singh, B. N., Mathew, S. \& ANand, N. (1958). The role of Aerobacter sp., Escherichia coli and certain amino acids in the excystment of Schizopyrenus russelli. Journal of General Microbiology I9, I04-I II.

Singh, B. N., Datta, T. \& DutTa, G. P. (1971). Factors inducing excystation in free-living amoeba. Indian Journal of Experimental Biology 9, 350-357.

Singh, B. N., SAXENa, U. \& Iyer, S. S. (1965). Production of viable sterile cysts of free-living amoeba and role of bacteria. on excystment. Indian Journal of Experimental Biology 3, 1 10-1 12.

Tomlinson, G. (1963). Mechanism of cyst wall formation in Acanthamoeba sp. Dissertation Abstracts 23, 3452 .

Tombinson, G. (1967). The glyoxylate pathway in Acanthamoeba sp. Journal of Protozoology 14, II4-I16.

TOMLInson, G. \& JonEs, E. A. (1962). Isolation of cellulose from the cyst wall of a soil amoeba. Biochimica et biophysica acta 63, 194-200. 